\title{
Comportement des sols fins compactés à l'humidification. Apport d'un modèle de microstructure
}

\author{
V. FERBER \\ J.-C. AURIOL \\ Route de Boulaye \\ BP 4129
}

Laboratoire centrad des ponts et chaussées

44341 Bouguenais Cedex valery. Ferber@ilcpc.fi jean-claude.aurioldcpc. fs

Y.J. CUI

CERMES-ENPC 6 et 8 , avenue Blaise-Pascal Gité Descartes

Champs-sur-Marne 77455 Mame-la-Vallée Gedex 2, cui@cermes.enpc.f

\section{J.-P. MAGNAN}

Laboratoire certral des ponts et chaussées

58 , bd Lefebvre

75732 Paris Cedex 15 jean-pierre.magnan@ilcpc.fr
Les déformations des sols fins dues aux variations d'état hydrique causent de nombreux dommages aux structures et ouvrages du génie civil, et on peut encore déplorer цr deficit méthodologique permettant leur prévision. Dans le cadre des recherches visant à optimiser l'emploi dies sols fins dans les remblais routiers et ferroviaires. l'étude présentée avait pour objectif de proposer une méthodologie d'étude destinée ả évaluer l'influence de la nature et de l'état initial des sols compactés sur les riscues de désordres par humidification.

Pour aborder cette guestion, un modèle de microstructure reposant sur l'organisation de la fraction argileuse en agrégats a été défini. Grâce aux đéveloppements technologiques récents en microscopie électronique à balayage et en porosimétrie par intrusion de mercure, les paramètres du modèle ont pu être formulés uniquement sur la base de paramètres gréotechniques conventionnels.

Ce modèle quantitatill de microstructure a été utilisé pour décrire le gonflement libre et les déformations par humidification sous contrainte, en étudiant l'influence du volume des vides inter-agrégats initial. Cette approche a permis de décrire quantitativement les évolutions de la microstructure causées par l'humidification, et de mettre au point une méthodologie d'étude des sols compactés dans la perspective de leur réutilisation dans des remblais en contexte délicat izone inondable, remblais de grande hauteur....).

Mots-cles: argile, microstructure, agrégats, gonflement, compactage, remblais, minéralogie, physico-chimie.

\section{Sensitivity of compacted fine-grained soils to wetting. Contribution of a microstructural model}

The deformations of fine-grained soils due to water content variations are responsible for mary darnages to civil engineering structures. In the framework of a research program aiming at optimising soil uses in roded and railways embankments, the present study was aimed at evaluating the influence of soil nature and intial conditions on the risk of etisorclers due to wetting.

This question was analysed by defining a microstructural model, based on the organisation of the clay fraction in aggregates. Thanks to recent technological developments in scaming electron microscopy and mercury intrusion porosimetry, fundamentals hypothesis were suggested in order to determine the model parameters from conventional geotechnical parameters. 
This quantitative microstructural model was used to describe the free swel] deformations and cfeformations due to wetting under vertical stress, by sturying the influence of initial inter-aggregate volume. Thanks to the microstructural model, the changes in intra-aggregate and inter-aggregate volumes were quantified and a new inethodology was developed for the design of roads and raitways embankrnents in sensitlye contexts (areas liable to flooding, high embankments...).

Key words : clay, microstruture, aggregates, swelling, cornpaction. embarkments, mineralogy, physiconchenistry.

\section{Introduction}

Les variations d'ëtat hydrique dans les sols fins sont à l'origine de déformations qui peuvent causer des dommages importants 5 les structures telles que les habitations, les ouvrages d'art, les chaussées ou les voies ferrées. Ces modifications de l'état hydrique du massif de sol résultent des variations météorologiques et peuvent atteindre une amplitude particulièrement importante dans les périodes exceptonnelles, causant alors des desordres pouvant renette en cause l'usage mềme des structures.

Les déformations des sols fins par séchage et humidification ont fait l'objet de nombreuses recherches, depuis plusieurs décennies, ce qui a permis de mettre en évidence trois points fondamentaux:

- les principaux paramètres quí gouvernent l'amplítude des déformations par séchage-humidification sont essentiellement la nature du sol (Seed et al., 1962), sa teneur en eau et sa masse volumique à l'état initial (Holtz et Gibbs, 1956), l'état de contrąinte appliqué pendant les sollicitations hydriques (Serratrice et Soyez. 1996) et enfin l'amplitude des sollicitations hydriques, c'est-à-dire l’amplitude des succions appliquées ali sol (Alonso et al., 1999):

- Jes déformations par variation d'état hydrique sont caractérisées par une composante réversible et une composante irréversible. Ainsi, à l'occasion d'ur cycle de séchage-humidification, le retrait dû̉ à la pase de dessiccation est partiellement compensé par le gonflement dû̉ à l'humidification grâce à la composante réversible du phénomène, mais on observe cependant une accumulation des déformations avec le nombre de cycles, mettanten évidence la composante irréversible (Alonso et al., 1999):

- la microstructure du sol, c'est-à-dire l'organisation de ses particules à l'échelle élémentaire, paraît avoir une influence swr les déformations (Cens et al., 1995) mais elle reste encore difficile a décrire quantitativement.

Ainsi, malgré les avancées dans la description de ces phênomènes, l'influence des différents paramètres gouvernant la deformation reste encore mal quantifiée et les méthodes de calcul et de prévision proposées dans la littérature sont basées soit sur des considérations empiriques dont la portée est. par nature, linnitêe (Derriche et Kebaili, 1998), soit sur des modèles de comportement reposant sur de nombreux paranètres dont la détermination est complexe et peu compatible avec la pratique courante de la géotechrique (Alonso et al., 1999).

Dans le cadre de la recherche présentée ici, qui porte sur le comportement des rembiais routiers et ferroviaires, la question des défomations par variations d'état hydrique a été abordée avec l'objectif de propo- ser une méthode de prévision des déformations pan humidification. L'idée sous-jacente est qu"une humidification complète constituerait la sollicitation la plus préjudiciable à un sol compacté. Cette hypothèse peut être discutée mais elle permet de se concentrer dans un premier temps seulement sur les conséquences de l'humidification.

La recherche d'une mëthode de prévision a corduit à mettre au point un modèle de microstructure qui sera presenté dans un premier temps. Lapplication de ce modele a l'interprétation des essais d'humidification sera présentée ensuite, dume part, pour les essais sous faible contrainte (gonflement libre) et, d'autre part. pour les essais d'humidification sous contrainte variable. Les interprétations serunt ensuite confrontées à des observations de la microstructure du sol pour evaluer la pertinence du modèle.

\section{2}

\section{Un modèle de microstructure des sols fins compactés}

\section{9}

\section{Quelques traits communs à la microstructure des sols fins compactés}

La microstructure des sols a fait l'objet de nombreuses recherches au cours des dernières décenries, aussi bien dans le domaine de la géotechnique que celui des sciences du sol. Depuis les travaux précurseurs de Lambe (1958), les connaissances sur l"inftuence des propriétés de nafure el d'état des sols sur la microsturture ont progressé de manière remarquable [Diamond, 1969; Tessier, 1984; Benett et Hulbem, 1985; Bruand et Prost, 1997 : Delage et al., 19961, jusqu'à sa modélisation par loutil mathématique des fractales (Bird et Perries, 2003). D'une manière simplifiée, ces différentes observations sur la microstructure et les propriétés physico-chimigues des sols fins compactés permettent de faire ressortir trois points esserutiels:

- le premier point est la différence fondamentale entre les particules argileuses et les particules non argileuses. Bien que la nature minéralogique des particules argileuses puisse être très variée, leurs propriétés physico-chimiques (surface spécifiçue, capacité d'échange cationique) et lewr morphologie (dimension. forme) en font des particules à part dans le sol. Pour les sols ne contenant pas de matière organique, on peut ainsi considerer en première approximation les particules non argileuses comme des particules inertes. La caractérisation et la quantilication de la fraction argileuse sont donc un point essential de l'identilication d'un sol; 
- Ie deuxième point est l'organisation des particules argileuses en agrégats : les particules dites "élémentaires a (Tessier, 1984), de dimension micrométrique, se regroupent en a paquets g que l'on qualifiera de particules a primaires "Fig. 1). Les particules primaires se regroupent elles-mênes en plus gros agrégats qui se regroupent eux-mëmes selon le même mode, conférant ansi à la structure du sol un caractère fractal (Benett et Hulbert, 1986. Gimenez et al. 1997). Même si la nature minéralogique conduit à des morphologies de particules prithatres différentes, il nous semble que l'on peut définir la particule primaire, que nous appellerons if agregat $m$, comme la structure de plus petite dimension formée par le regroupement de particules élémentaires. Enfin, cette structuration en agrégats conduit è différencier des pores intra-agrégats, localisés dans la particule primaire, et des pores inter-agrécats, localisés entre les particules primaires. Les premiers sont de beaucoup plus petite dimension que les seconds;

- Ie troisieme point est la relation entre indice des vides et volume inter-agrégats : il a été montré que les particules primaires ne sont pas affectées par le processus de compactage, et que le volume des pores inter-agrégats diminue avec l'indice des vides (Delage et al., 1996; Wan et al., 1995).

Ces trois points méritent d'être complétés par une réflexion sur la localisation de l'eau dans un sol fin compacté non saturé. Dans ce type de sol, les succions les plus élevées sont dues à la composante d'adsorption des particules arcileuses, au sein des agrégats argileux. Par ailleurs, ta composante capillaire, qui dépend du rayon des pores, est logiquement plus forte au sein des agrégats que dans les pores inter-agrégats, qui sont de plus grande dimension. Ces elements suggèrent donc que l'eau doit être attirée préférentiellement au sein des agrégats et que c'est donc là sa localisation privilé giée dans un sol non saturé.

Ces hypotheses ont pu être confrontées à des observations au microscope électronique ả balayage (MEB) environnemental, outil qui permet d'observer un même échantillon à l'échelle microscopique au cours d'une humidification et/ou d'un séchage. Ces observations montrent que, lors de l'humidification d'un sol fin compacté intialement sec (Fig. 2a), l’eau (qui forme un film noir sur les images MEB) pénètre d"abord dans les agrégats argileux (Fig. 2b) et ne remplit les vides inter-agrégats que dans un second temps. Cest donc bien au sein des agrégats argileux que l'eau est attirée préférentiellement.

\section{0}

\section{Formulation du modèle}

Ces observations communes ì l'ensemble des sols fins compactés permettent de proposer une quantification simple des volumes des vides intra-et inter-agrégats, selon une approche déjà proposée pour étudier et modẻliser le retrait des sols [Braudeau, 1988; Boivin et al., 2004). En effet, si l'eau du sol est localisée au sein des agrégats, c'est que les vides inter-agrégats ne contiennent que de l'air. Si l'on suppose en outre que les agrégats sont saturés d'eau, le volume des vides intra-agrégats peut être assimilé au volume d'eau et le volume des vides inter-agrégats peut être assimilés au volume d'air. Ceci permet de définir.

- un indice des vides des agrégats, noté e $e_{\text {ag }}$ (é. 1), quí est le rapport entre le volume d'eau et le volume des particules solides :

- un indice des vides inter-agrégats, noté $\mathrm{e}_{\text {-ag }}$ (ég. 3). qui est le rapport entre le volume d'air et le volume des particules solides, mais qui est aussi la différence entre i’indice des vides global du sol et l'indice des vides des agrégats (éq. 2).

$$
\begin{aligned}
& \mathrm{e}_{\mathrm{ag}}=\frac{V_{w}}{V_{s}}=\frac{w \rho_{1}}{\rho_{w}} \\
& \mathrm{e}=\mathrm{e}_{\text {晒 }}+\mathrm{e}_{1-\mathrm{ag}}
\end{aligned}
$$

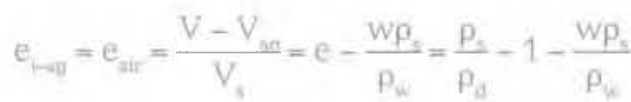

Selon ce modèle, l'indice des vides des agrégats

\section{Structure des particules primaires selon la nature minéralogique}

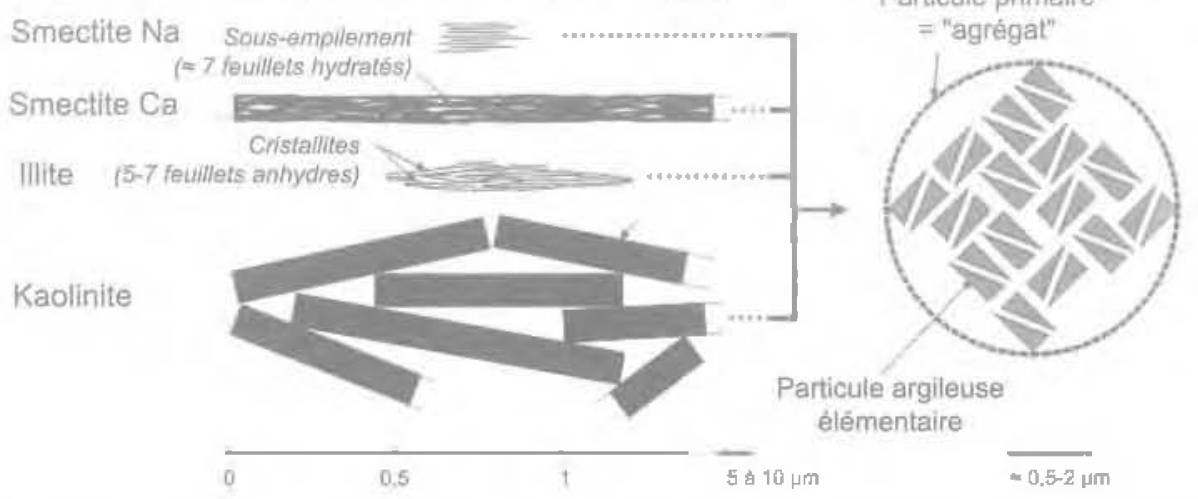

Représentation simplifiée des différentes formes de partícules primaires par un modèle générìue de particule primaire. ("Tessier, 1984). 

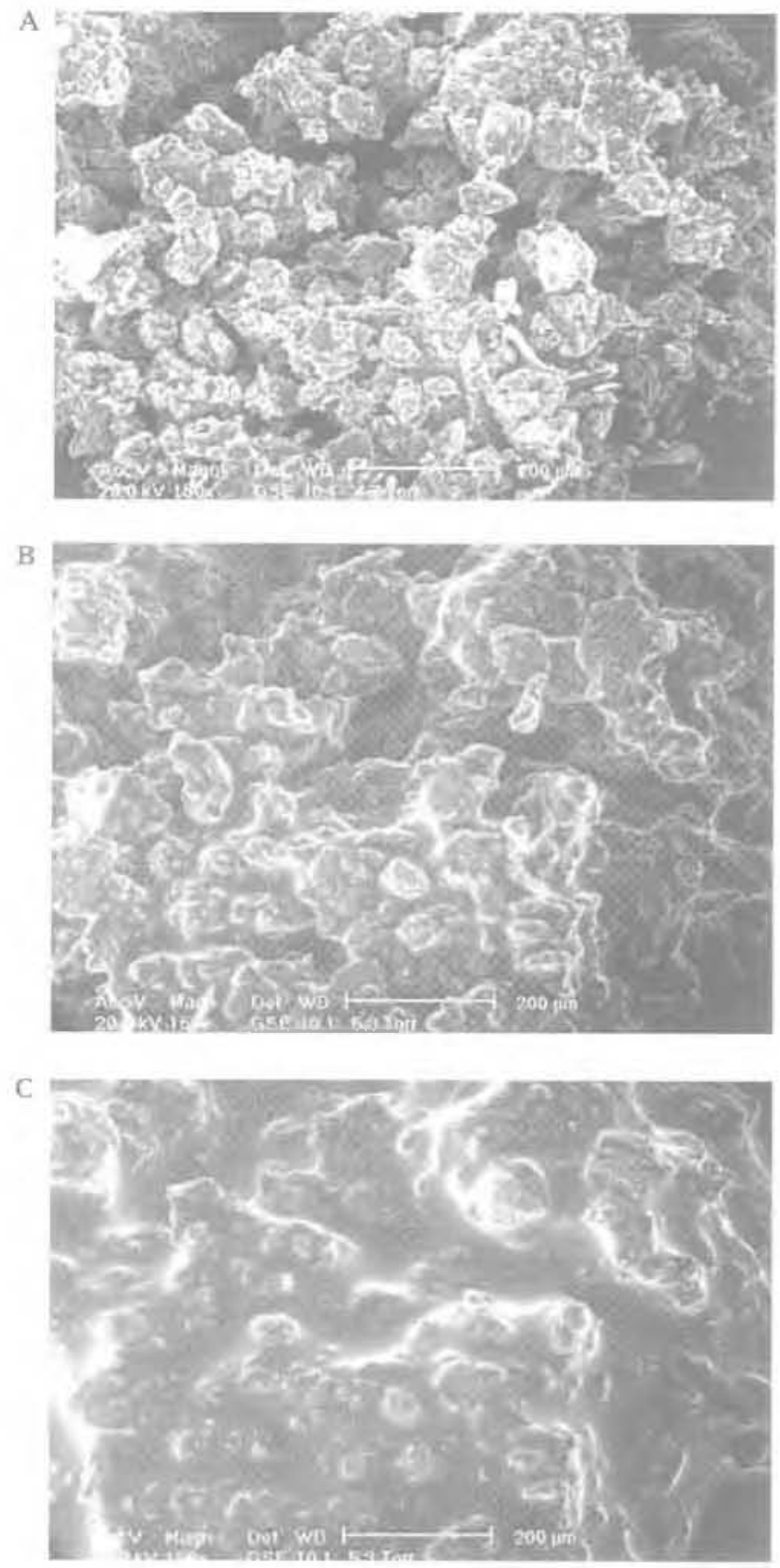

FG. 8 Observation de particules argileuses à différentes étapes (A : avant humidification. $B$ : après hydratation des agregats : $C$ : après remplissage des vides inter-agregats:

augmente linéairement avec la teneur en eau du sol et l’indice des vides inter-agrégats résulte de l'influence conjuguée de la teneur en eau et de l'indice des vides global du sol (Fig. 3).

\section{3}

\section{Étude du gonflement libre}

Le modèle de microstructure présenté dans le paragraphe précédent permet de quantifier deux paramètres microstructuraux du sol compacté non saturé. Dans la suite de cet article, on cherchera ì décrire l'évolution de ces paramètres au cours d'essais d'humi- dification, et dans un premiel tenups d'essais sous faible contrainte, appelès essais de a gonflement libre a.

\section{1}

\section{Procédure expérimentale}

Le principe d'un essai de gonfement libre, tel cue réalisé dans le cadre de cette recherche est de compacter un échantilon de sol dans min moule oedométrique. de le soumetre $\mathbf{a}$ une contrahte verticte de $3 \mathrm{kPa}$, d immerger la cellule cedométrique avec de l'eau dé-ionjsée et de suivre les défonmations dues à l'adsorption de l'eau par le sol, Le compactage a été réalisé au moyen d'une dame de compactage miniaturisée et la surface des échantillans a été égalisée au moven d"une règle à araser, de sorte que tous les échantillons avajent une hauteur initiale de $19 \mathrm{~mm}$ pour un diamètre de $70 \mathrm{~mm}$.

Dans cette étude, les échantillons ont été compactés à différentes masses volumiques sẻches initiales en faisant varier le nombre de coups de compactage. A chaque série d'essais correspond ainsi une même teneur en eau Initiale et six masses volumigues sèches initiales, en général La teneur an eau a été obtenue par un séchage préalable du matériau et par une humidification choisie. Les échantillons n'ont été compactés qu'après un minimum d'une journée de conservation du sol humiditié en sac etanche.

\section{Influence de l'état initial - cas de l'argile verte de Romainville}

L'étude de l'influence de l'état initial sur le gonflement libre a été réalisée sur de l'argile verte de Fomainville [Sannoisienj, prélevée prếs de Saint-Leu-la-Forêt dans le Val-d’Oise (Tableau I]. La fraction argileuse de ce sol, qui représente $67 \%$ de la masse des particules solides, est constituếe en majorité d'illite ( $75 \%$ ) et d'une proportion non négligeable de smectites (10\%).

Les échantillons ont étể compactés à six teneurs en eau intiales dont cing étaient inférieures à la teneur en eau de loptimum Proctor normal (Fig. 41. En reportant f'indice des vides global ces echantillons en fonction de l’indice des vides inter-aglégats initial, c'est-à-dire après compactage et avant humidification (Fig. 4), on constate

- qu'une reletion linéaire apparaìt entre les cieux paramètres pour les échantillons ayant la mềme teneur en eau initiale;

que les séries correspondant aux trois teneurs en eau les plus élevées semblent se superposer dans ce diagramme alors qu'un décalage apparaît pour les séries dont la teneur en eau initiale était plus frable. De plus. ces séries sont d'autarat plus éloignées des séries " huñides ì que leur teneur en ea initiale est faible.

Ges rếsultats laissent å penser que le gonflement libre d’un sol est plutôt bien caractérisé par son état final, qui paraît pouvoir être décrit par une relation linéaire entre l'indice des vides inter-agrégats initial et l'indice des vides global après gonflement. Dans cette perspective, le fait que les échantillons les plus secs présentent un décalage suggère cependant que leur état initial serait mal caractérisé par le modèle de nicrostructure jnitialement proposé. En particulier, le 

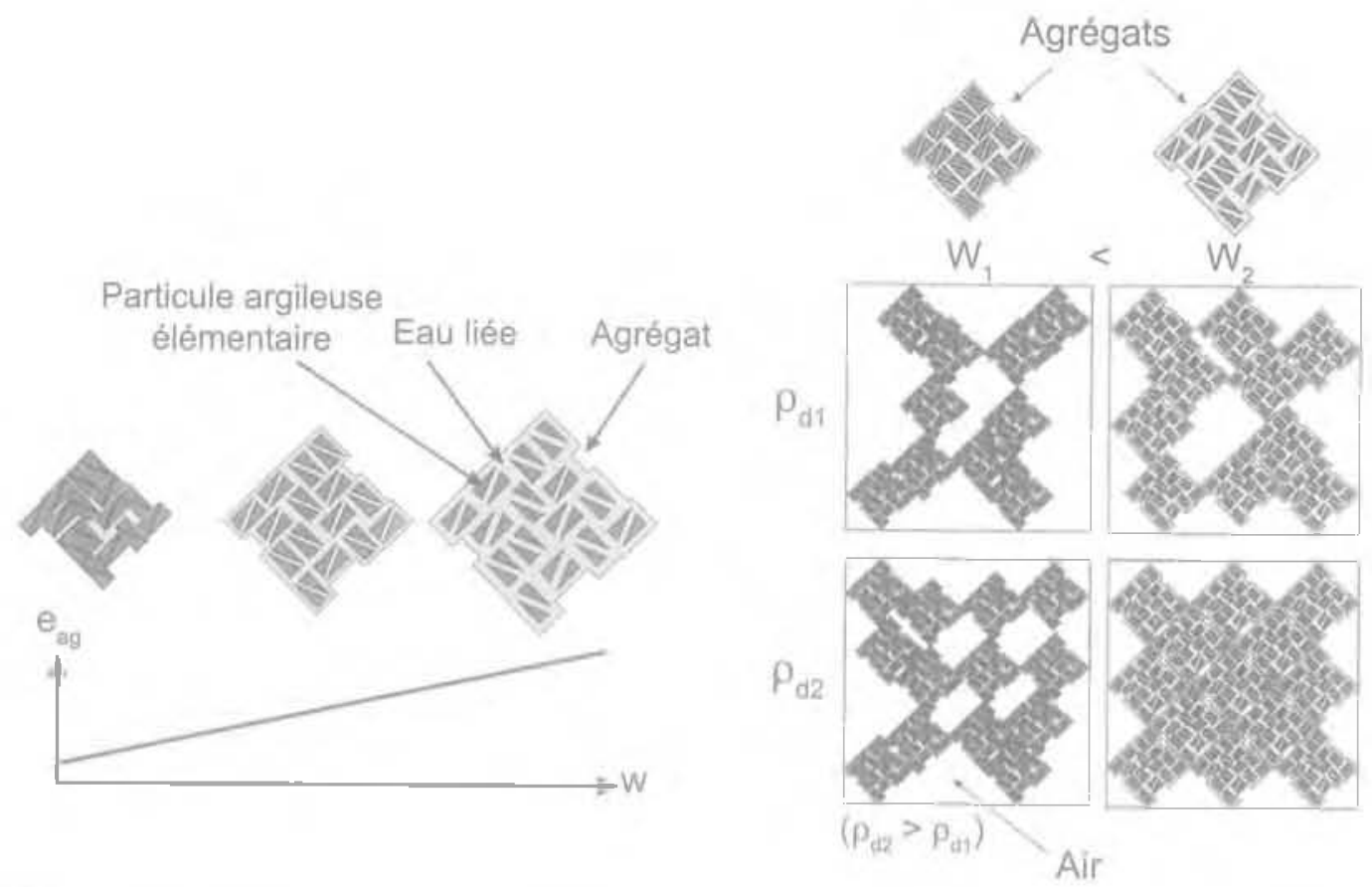

ตG. 3 Illıstration schématique de l’ỉnfluence conjuguée de lą teneur en eau et de la masse volumicque sèche sur ta microstructure.

тAsıu Propriétés géotechniques de l'argile verte de Romainville. Geotechnicail characterisation of Ronainville green clay.

\begin{tabular}{|c|c|c|c|c|c|c|c|}
\hline$W_{1} \mid(\%)$ & $t_{n}$ & Gyis & $\begin{array}{c}\mathrm{P}^{\mathrm{B}} \\
\left(\mathrm{Mg}^{\prime} / \mathrm{m}^{4}\right)\end{array}$ & $W_{6}$ & $\begin{array}{l}\text { pd, opN } \\
\left(\mathrm{Mg} / \mathrm{m}^{3}\right)\end{array}$ & $\begin{array}{l}\text { Valeur de bleu } \\
(\mathrm{g} / 100 \mathrm{~g})\end{array}$ & $\begin{array}{c}\text { CEC } \\
\text { (cmal + ka) }\end{array}$ \\
\hline 70,3 & 39 & 67 & 2,76 & 26 & 1,55 & 5,4 & 18,9 \\
\hline
\end{tabular}

fait de supposer que les agrégats sont saturés à l'état initia! pourrait introduire un biajs expliquant ce décalage.

Le décalage semble apparaître à partir d'une teneur en eau initiale d"environ $20 \%$, que l"on appellera " teneur en eau critique o (notée $\left.w_{\text {crituve }}\right)$. On propose de modifier légèrement l'équation 1 si la teneur en eau initiale est inférieure. Dans ce cas, on supposera que les vides intua-agrégats sont remplis d'eau et d'air mais que leur volume re varie pas tant que la teneur en eau reste inférieure à la teneur en eau critique. Le volume des vides serait alors égal au volume d'eau que contiendrait le sol à la teneur en eau critique (éc. 4). On en déduit l'indice des vides inter-agrégats grầce à l'équátion 2. Les équations 1 ef 3 resterajent valables pour les teneurs en eau supérieures à la teneur en eau critique.

$$
\text { Si w< w willique } \quad e_{\text {ad }}=\frac{w_{\text {critigue }} \rho_{s}}{\rho_{w}}
$$

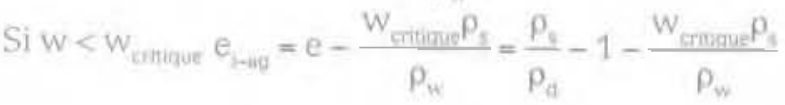

La représentation de l’indice des vides global après gonflement libre en fonction de ce nouvel indice des vides inter-agrégats conduit alors à éliminer le décalage entre les séries d'essais (Fig. 5). En calculant l'ordonnée à l'origine et la pente des droites de régression pour les différentes séries d'essais, on constate que l'ordonnée à l'origine des droites à l'état final est relativement constante et indépendante de la teneur en eau initiale. alors que la pente a lendance à diminuer lorsque la teneur en eau initiale est plus faible (Fig. 6).

\section{3}

\section{Interprétation microstructurale}

La relation linéaire observée entre l'indice des vides global après gonflement libre et l'indice des vides inter-agrégats initial permet de caractériser le comportement à l'humidification grâce à deux paramètres qu sont la pente et l'ordonnée à l'origine des droites de régression. On cherchere ici à préciser la signification de la relation linéaire et de ses paramètres.

Concernant l'ordonnée à l'origine des droites, on peut considérer qu'elle correspondrait à l'indice des vides après gonflement libre d'un échantillon dont l'indice des vicles inter-agrégats initial serait nul. Autrement dit, l'ondonnée à l'origine pourrait constituer l'indice des vides après gonflement libre des agrégats. Cette interprétation est assez coherente avec le fait que ce paramètre ne dépend ni de l'indice des vides initia! ni de la teneur en eau initiale. Ce serait donc un paramètre intrinsèque du sol décrivant la capacité totale d'adsorption d'eau de ses agrégats.

Ĺinterprétation de la pente des droites impose ur retour sur la description de l'état initial. En effet. l'équation 3 indique qu'il existe, par défirition. une relation linéaire entre l'indice des vides inter-agrégats initial et l'indice des vides global initial. Cette relation 


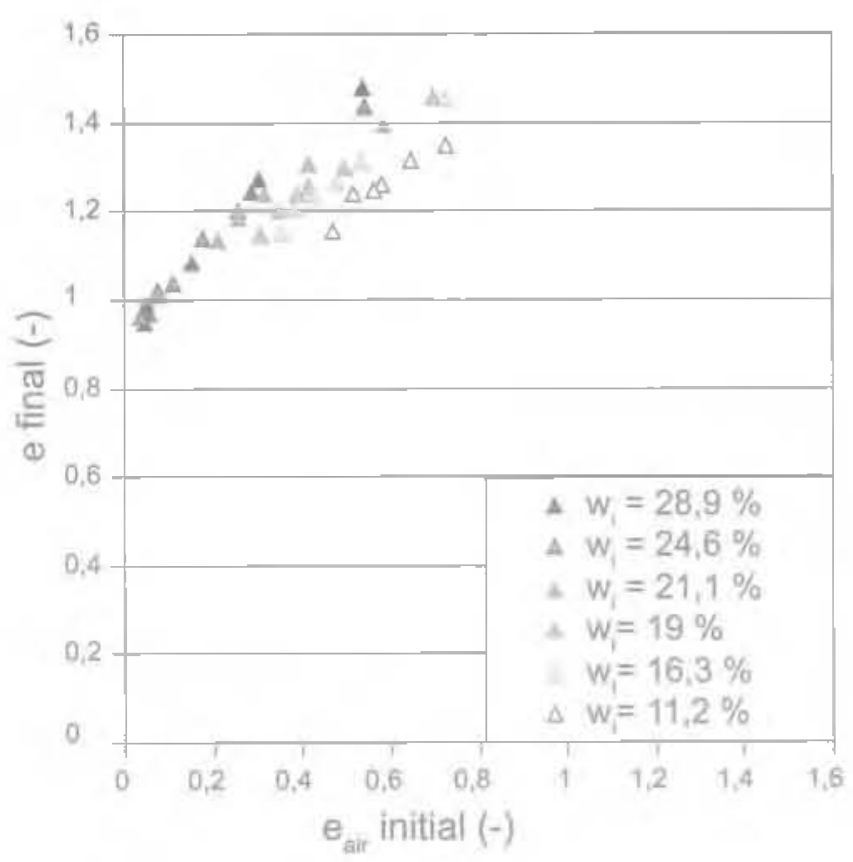

Fis. A Influence de l'indice d'air initial sur I'indice des vides apres gonflement libre (argile verte de Fomanvilie].

ilustre simplement le fait, gu'aे teneur en eau initiale constante, l'indice des vides inter-agrégats augmente avec l'indice des vides global et çue la relation linéaire entre ces deux paramètres a une pente de 1 (Fig. 7). Après gonflement libre, l'indice des vides global est toujour's la somme de l'indice des vides des agrégats et de J'indice des vides inter-agrégats (éq. 6) mais les résultats expérimentaux indiquent, de plus, quili existe une relation linéaire entre l'indice des vides final et l'indice des vides inter-agrégats initial (éc. 7). Si l'on admet que l'ordonnée à l'origine, notée ici i j, est égale àindice des vides des agrégats final, noté e comme I'interprétation précédente le suggère, la pente, notée

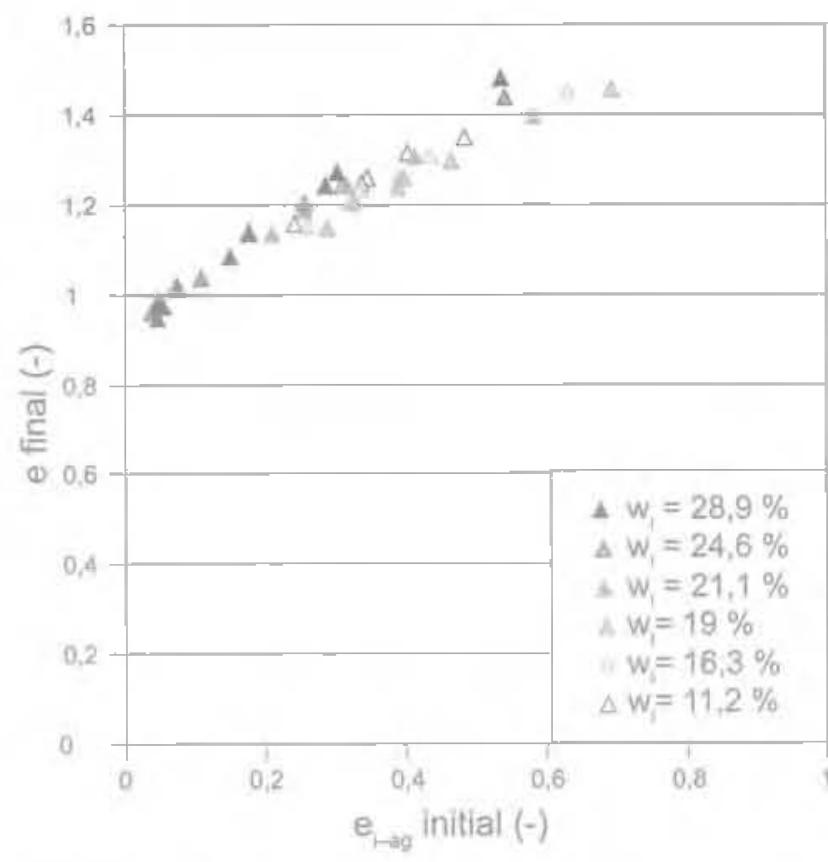

16.5 Influence de l'indice des vides inter-acirégats initial sur l'indice des vides après gonflement libre [argile verte de Homanville].

ici $\alpha$, correspond alors at rapport entre l'indice des vides inter-agrégats final et lindice des vides interagrégats initial (éc. 8) :

$$
\begin{aligned}
& e_{\mu}=e_{j-t g, t}+e_{\text {egg } f} \\
& e_{t}=\alpha e_{\text {1-的, }}+\beta \\
& a=\frac{S_{\text {ast }}}{\text { Rem }}
\end{aligned}
$$

Ainsi, une pente inférieure à 1 indique que le gonflement libre a généré une diminution du volume des
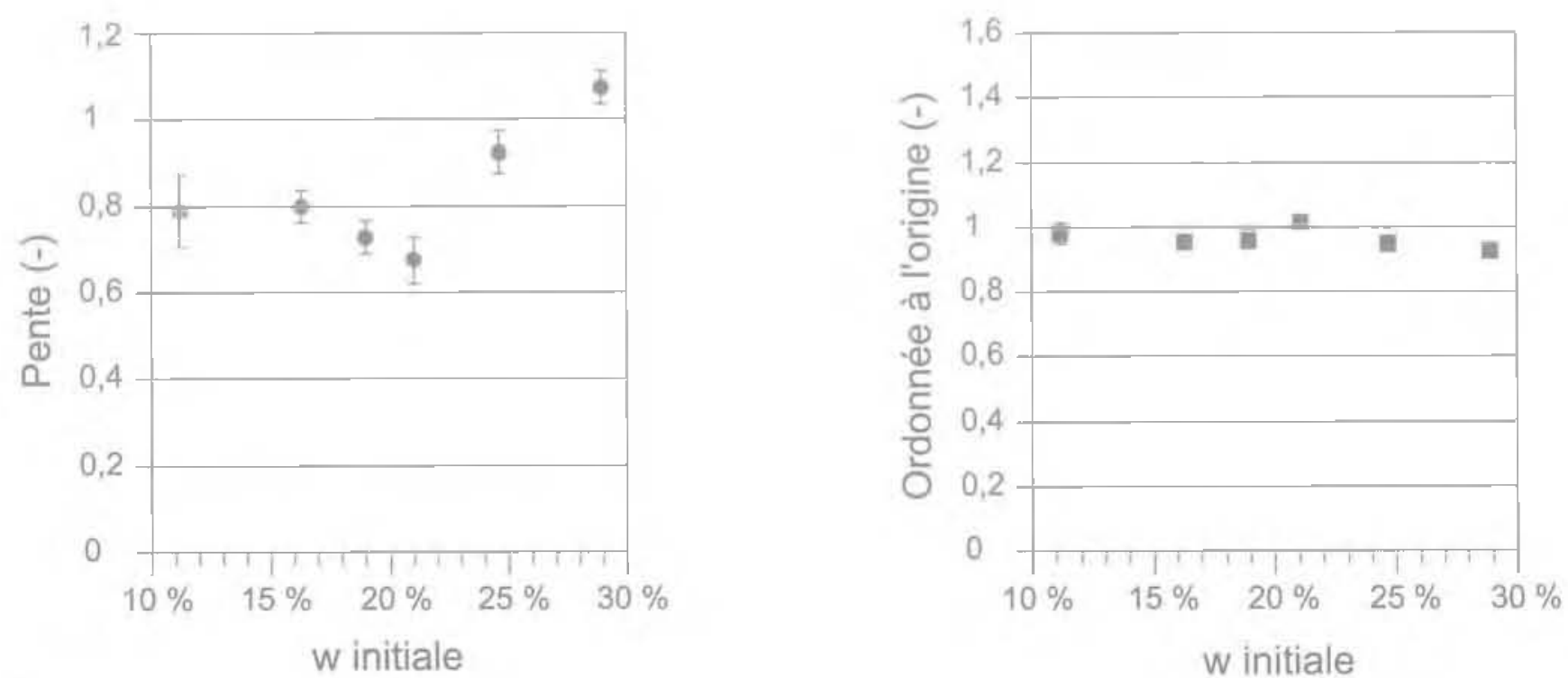

F6. Influence de la teneur en eau imitiale sur la pente et l'ordonnée à l'origìne des droites de régression linéaike calculés avec l'indice des vides inter-agrégats initial. 


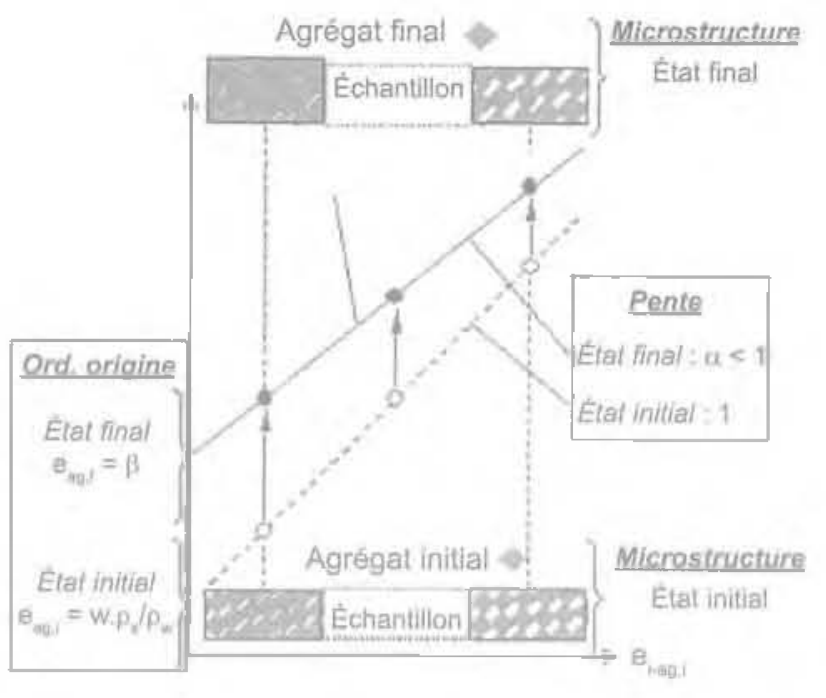

FG. 7 Représentation schématigue de l'interprétation des paramètres de l'état final dans le diagramme $\left(e_{h-1, !}, e\right)$.

vides inter-agrégats, et les résultats prẻsentés suggèrent que cette diminution est d'autant plus forte que la teneur en eau initiale est fafble (Fig. 6). Autrement dit, un échantillon de sol perd proportionnellement d"autant plus de vides inter"agrégats lors du gonflement libre quilil a été compacté à une faible teneur en eau, et ce, quel que soit son indice des vides initial.

Enfin, la relation linéaire indique que le pourcentage de volune des vides inter-agregats perdu au cours du gonflement est une constante, indépendante du volume initial des vides inter-agrégats. Aucune explication rigoureuse ã ce résultat surprenant n'a pu être proposée.

\section{4}

\section{Influence de la nature des sols}

La méthodologie d'étude et d'interprétation décrite précédemment a été appliquée à un panel de dix sols naturels allant du limon peu argileux â l'argile trés plastique. Poun chaque sol, des series d'essais de gonflement libre ont été réalisées à partir de teneurs en eau et de masses volumiques sèches initiales variées. Les résultats ont canfirmé qu'jl était nécessaire de prendre en compte le concept de terreur en eau critique, que la pente des régressions linéajes sur les essais à même teneur en eau initiale diminuait avec la teneur en eau initiale et que l'ordonnée à l'origine de ces régressions était quasiment insensible à la teneur en eau initiale.

Cette insensibilité de l'ordonnée à l'origine à l'état initial confère à ce paramètre un caractère intrinsèque, qui lui permettrait de refléter" le potentiel d'adsorption d'eau des agrégats du sol, indépendamment de leur état hydrique et de leur agencement intial. Pour vérìfier cette idée, ce paramètre a été confronté à la limite de liquidité, paramètre d'identification du sol dont on peut aussi attendre a priori un caractère intrinsèque. Étant donné que la limite de liquidité est mesurée sur la fraction 0-400 micrométres du sol et que les essais de gonflement libre ont été réalisés sur la fraction 0-2 millimètres, la confrontation a été réalisée avec une limite de licuidité corrigée par le pourcentage de particules de diamètre inférieur à 400 micromètres, noté $\mathrm{C}_{\text {qum }}$ (Fig. 8).

Cette confrontation révèle effectivement une corrélation intêressante entre ces deux paramètres pour les sols naturels, ce qui tend à confirmer que l'ordonnée à l'origine das droites de régression pourmit caractériser le potentiel d'adsorption d'eau des agrégats. Ce potentiel d'adsorption à l'échelle des agrégats pourrait en outre être estimé de manière assez pertinente par la limite de liquiditể corrigée.

On doit ajouter que la corrélation s'est, en revanche, avérée insuffisante pour une argile de carrière (kaolinite Speswhite), ce qui a pu être expliqué par la forte proportion de cations adsorbés monovalents et en particulier de sodium. A priori, la corrélation ne parailt donc pertinente que pour les sols contenant des argiles à tendance plutôt calcique, ce qui représente toutefois la très grande majorité des sols nattrels. Cecj devra être étudié sur un parel encore plus large de sols, comprenant notamment des sols à tendance sodique.

\section{5}

\section{Conclusion pour le gonflement libre}

Une approche microstructurale semble donc permettre de distinguer les différents phénomènes à l"origine des déformations par humidification dans les sols fins compactés. En particulier, il apparait que le gonflement libre meșré à l'échelle macroscopique résulte d'un gonflement des agrégats, qui semble pouvoir ètre prévisible grâce à la limite de liquidité corrigée. et d'une réorganisation des agrégats, qui génère une diminution des vides inter-agrégats. Cette interprétation va être confrontếe de nouvelles données expèrjmentales dans la partie suivante, consacrée à l"humidjfication sous contrainte.

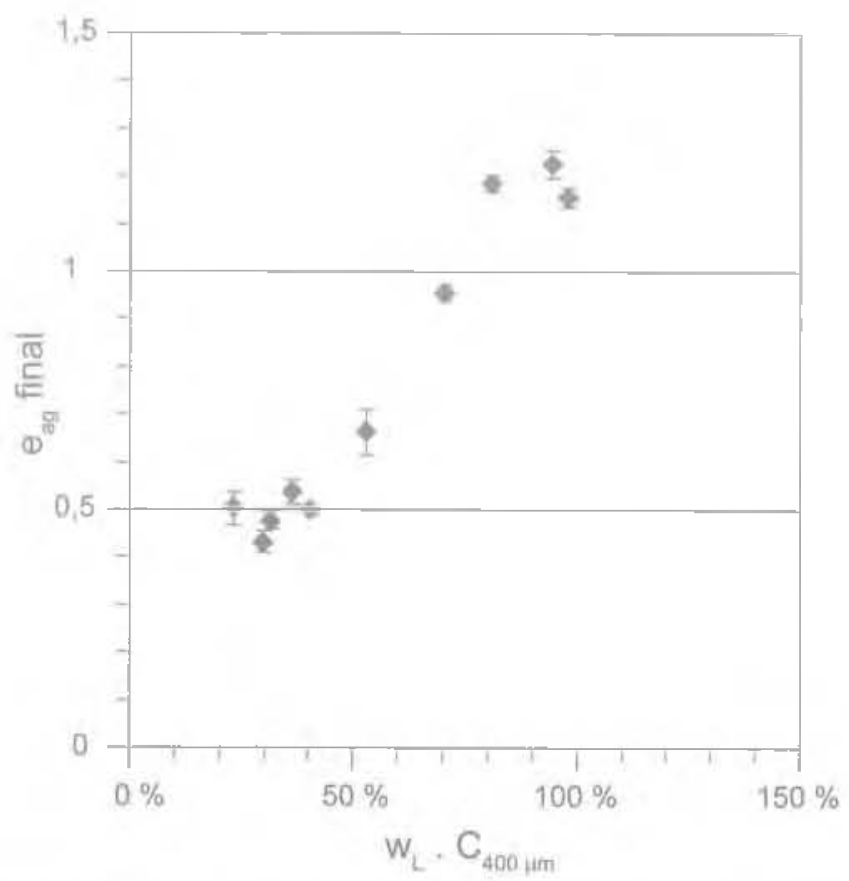

Tt. $\theta$ Influence de la teneur en eau injtiale cortigée des vides des agrégats aprés gonflement libre de dix sols naturels. 


\section{Étude de l'humidification sous contrainte}

\section{1}

\section{Procédure expérimentale}

La procédure expérimentale est quasiment identi que à celle utilisêe pour les essais de gonflement libre (cf. \$ 3.1). Le sol est compacté dans un moule oedométrique au moyen d une dame de compactage minia turisée, sa surface est égalisée. puis il est soumis à la contrainte verticale choisie. Ce chargement mécanique à teneur en eau supposée constante se fait relativement rapidernent pour les contraintes inférieures à $500 \mathrm{kPa}$ et peut prendre une joumee environ pour les plus fortes contraintes. La contrainte maximale utilisée dans cette étude est de $1400 \mathrm{kPa}$.

Une fois que les tassements ả tereur en eat constante sont stabilisés, la cellule aedamétrique est remplie d'eau délonisée et les déformations sont suivies jusçu'à stabilisation.

Comme pour le gonflement libre, ce protocole a été appliqué à des sêries d'échantillons compactés à différentes masses volumigues sêches et è mể teneut" en. eatu initiale.

Létude du comportement ả l'humidification sous contrainte a été limitée à deux sols (Tableau II) représentant, pour l'argile AvA34, les sols très argileux et, pour le limon LGod, les sols limoneux moyennement argileux. L’argile AvA34 (Abien, Secondaire) a êté prélevée sur le chantier de l'autoroute A34 cui relie Charleville-Mézières à Rethel (Ardennes). Sa fraction arglleuse est constituée de $60 \%$ de smectites et de $40 \%$ d'illite. Le limon LGod a été prélevé sur le chantier de la déviation de Goderville (Seme-Martime) et sa fraction argileuse est constituee à $60 \%$ d'illite et à $40 \%$ de kaolinite

\section{2}

\section{Comportement d'une argile (AvA34)}

Lindice des vides après humicification de l'argile AvA 34 a été reporté en fonction de l'indice des vides inter-agrégats initial (Fig. 9). Seule une teneur en eau intiale, égale à $22 \%$, a été étudiée.

On peut observer (Fig. 9) que, à contrainte verticale constante, l'indice des vides après humidification présente une relation linéaire avec l'indice des vides interagrégats initial, comme cela a été observé pour le gonflement libre. On constate que l'ordonnée à l'origine et la pente des droites de régression dimintient lorsque la contrainte verticale augmente, selon une lol semi- logarithmique (Fig. 10). En reprenant Jinterprötation proposée précédemment pour ces deux paramètres (cf. \$3.3), on peut déduire de ces résultats que :

- la pente diminuant lorsque la contrainte verticale augmente, l'humidification sous contrainte génère une diminution du volume des vides inter-agregats d"autant plus forte que la contrainte verticale est élevée. On trouve même une valeur négative de lá pente sous $1400 \mathrm{kPa}$, ce qui révèle peut-être les limites cu modèle en teme de précision. En effet, or ne peut concevoir que le volume des vides inter-agrégats soit négatif :

- l'ordonnée á l'origine des droites de régression, qui représenterait l'indice des vides des agrégats après humidification, diminue aussi lorsque la contrainte augmente. Cependant, on peut constater" qu'il reste supérieur à l'indice des vides des agrégats initial pour les contraintes inférieures a $800 \mathrm{kPa}$. Ce n'est donc qu'au-delả de $800 \mathrm{kPa}$ que la contrainte verticale empếche les agrégats de gonfler.

Ainsi, lors de l'humidification sous contrainte de cette argile, les tassements observés lors de l'humidification serakent essentiellement dus à la diminution du volume inter-agrégats, alors que les agrégats eux-mêmes présenteraient un gonflement pout les contraintes infêrieures à $800 \mathrm{kPa}$.

\section{3}

\section{Comportement d'un limon}

Le même protocole appliqué à un límon (linnon de Goderville) sous une contrainte verticale de $100 \mathrm{kPa}$ conduit à une toute autre constatation. En effet, on n'observe plus de relation linéalre entre l"indice des vides après humidification et lindice des vides inter-

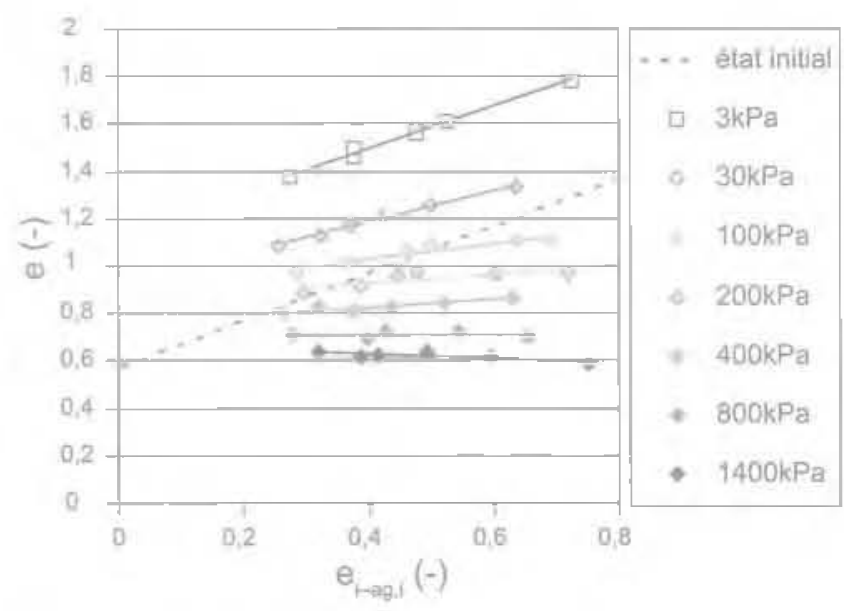

FIG. Influence de l'indice des vides interagrégats initial sur l'indice des vides après huraidification sous differentes contraintes verticales (argile AvA34).

TAalEAL II Propriêtés géotechniques de l'argile AvA34 et du limon de Goderville LGod. Geotechnical characterisation of AvA34 clay and Lgod Goderville silt.

\begin{tabular}{|c|c|c|c|c|c|c|c|c|}
\hline & $W_{1}(w)$ & $I_{\psi}$ & S\%] & $\stackrel{\rho}{\left(\mathrm{Mg}_{\mathrm{g}}\left(\mathrm{m}^{3}\right)\right.}$ & $\begin{array}{l}w_{\text {(sete }} \\
\text { (\%) }\end{array}$ & $\begin{array}{l}\mathrm{P}_{\mathrm{e}} \\
{\left[\mathrm{M} / \mathrm{m}^{3}\right]}\end{array}$ & $\begin{array}{l}\text { Valeur } \\
\text { de tsteu } \\
\text { (a/.100 gl }\end{array}$ & $\begin{array}{c}\mathrm{CEC} \\
\text { [crmol }+\mathrm{kg}]\end{array}$ \\
\hline AvA34 & 98 & 61 & 66 & 2,71 & 28 & 1,45 & $10, \overline{7}$ & 41,2 \\
\hline LGod & 41 & 19 & 35 & 2,67 & 17 & 1.75 & 3.5 & 12,5 \\
\hline
\end{tabular}



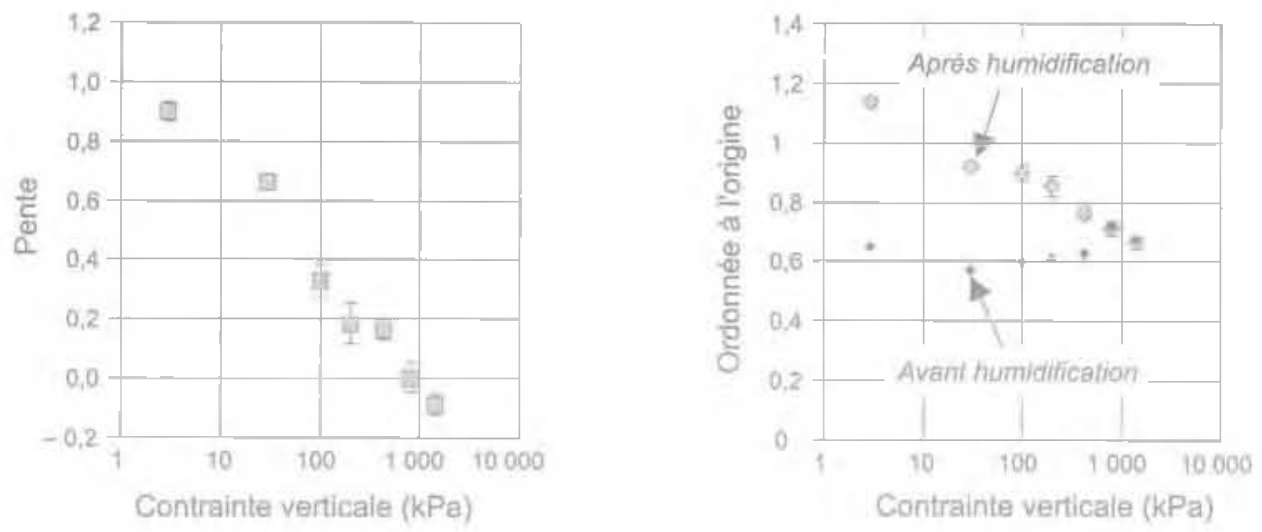

nG. Io Influence de la contrainte verticte sur l’ordonnée à l'origine el la pente des droftes de régression (AvA34).

agrégats initial (Fig. 11). Par contre, on peut constater que les échantillons les plus compactés ne présentent que de faibles déformations, alors que les échantillons les moins compactés (fort indice des vides inter-agrégats initial') subissent un tassement d'autant plus êvevée qu'ils sont peu denses à l'état initial.

Ce résultat doit être accompagné d'une autre observation sur l'état final des échantillons. En effet, or peut observer que, lorsque les échantillons tassent du fait de l'humidification, leur indice des vides final atteint une valeur apparemment constante, qui ne dépend ni de l'indice des vides inter-agrégats intial, ni de la teneur en eau initiale (Fig. 111. On appellera cet indice des vides undices des vides aprés effondrement $n$. en référence au phénomène qui semble conduire au tassement par humidification. Ce constat a pu être fait pour les essais sous toutes les contraintes supérieures à $100 \mathrm{kPa}$ et il apparaît que cet indice des vides après effondrement diminue lorsque la contrainte augmente selon une loi semi-logarithmique (Fig. 12). Il a pu être montré que cette courbe n'était autre que la courbe de compression vierge cedométrique du sol saturé.

D'un point de vue pratiçue, ce résultat montre que le compactage du limon $95 \%$ de sa masse volumique sèche à l'optimum Proctor normal devrait permettre de se prémunir des déformations par humidification pour une hauteur de remblai de dix mètres, correspondant ả
$200 \mathrm{kPa}$ (Fig. 12). Ceci paraît relativement cohérent en ordre de grandeur avec les prescriptions de compactage pour les remblais courants (SETRA-LCPC. 1992). Il montre aussi que cette mëthode pourrait pernetire de proposer des prescriptions de compactage adaptées à la hauteur de l'ouvrage étudié et éventueltement a nature du ratérial.

\section{4}

\section{Conclusion pour l'humidification sous contrainte}

Ainsi, l'indice des vides des échantillons inguffisamment compactés de limon ne dépend que de la nature du sol et de la contrainte verticale. Ceci confijme l'intérêt de la méthode du double-cedomètre (Lawton et al., 1989) pour la prévision des déformations par humidjfication des sols moyennement argileux. On a vu précédemment que les phénomènes êtaient en revanche plus complexes pour les sols très argileux.

Cette différence fondamentale de comportement pourTait provenir de la différence de texture entre ces sols, et en particulier de la différence de pourcentage d'argile. En effet, l'arcille AvA34 est un sol contenant près de $70 \%$ de particules argileuses et son comportement est donc quasiment exclusivenent gouverie par cette f̈raction. En revanche, le limon ne contient que $35 \%$ de parti-
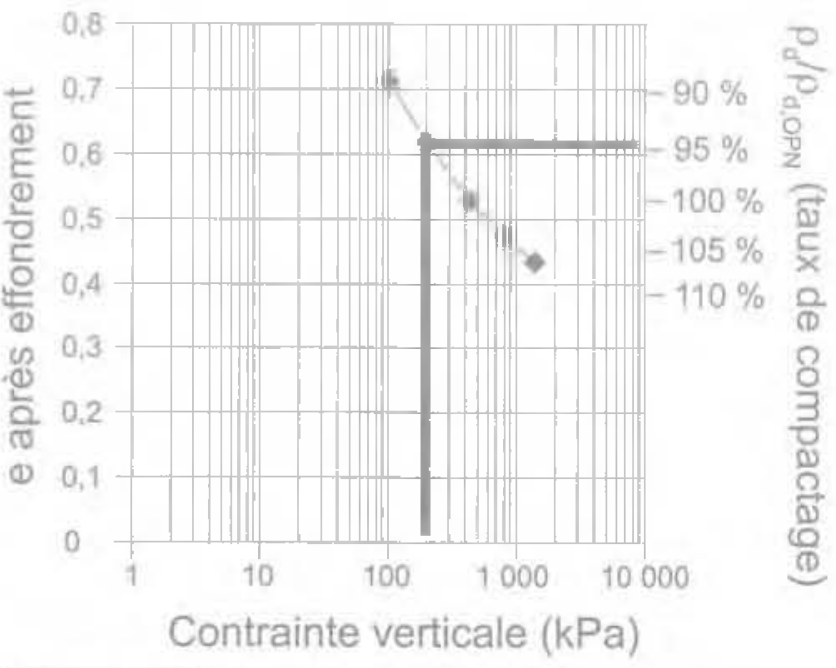

HG. 12 Influence de la contrajute verlicale sur ]'irdice des vides après effondrement (limon LGod). 
cules argileuses, et son comportement est probablement affecté conjointernent par les déformations de sa fraction argileuse et раг les interactions frottantes entre les partcules non argileuses. C'est ce qui expliquerait l'absence de relation linéaire entre l'indice des vides et l'indice des vides inter-agrégats pour le limon.

\section{5}

\section{Confrontation aux porosimétries par intrusion de mercure}

Pour évaluer la pertinence des hypotheses du modèle de microstructure et des interprétations proposées qui en découlent, des échantillons d'argile AvA34 ont été préparés spécialement pour des analyses par porosimétrie au mercule. Cette technique expérinentale permet de déterminer la distribution des volumes de pores d'un échantillon de matérieu en fonction de Ieur dimension, en forçant l'injection d'un liquide non mouillant (le mercure en l'occurrence) ia différentes pressions. En utilisant la loi de Jurin-Laplace (Pellerin, 1980), les pressions peuvent être converties en diamètre de pores. Linterprétation de ces essais doit intégrer la petite dimension des échantillons (guelques grammes) et les effets de leur séchage, même si celui-ci est réalisé par lyophilisation (Delage et ai., 1996), ce qui est le cas des échantillons étudiés icî.

Dans un premier temps, les distributions de tailles de pores ont été mesurées sur trois échantillons d'argile AvA34 compactés à une même teneur en eau (environ $22 \%$ ) mais a trois indices des vides différents (Fig. 13).

On peut observer la double structure de ce type de sol, avec des micropores de diamètre inférieur à 1 micromètre, et des macropores de diamètre supërieur à 10 micromètres. Les premiers correspondent probablement à ce qui a été appelé icl pores intra-agrëgats alors que les seconds correspondraient aux pores interagrégats. Comme l'avaient indiqué d'autres auteurs auparavant (Delage et al., 1996; Wan et al. 1995), les micropores ne semblent pas affectés par le cornpactage, qui conduit en revanche à une diminution du diamètre médian et du volume total des macropores.
Par ailleurs, deux échantillons faiblement compactés (indice des vides après compactage de 1,1) ont été sournis, pour le premier, à un gonflement libre et, pour le second, à une humidification sous $200 \mathrm{kPa}$, puis à un déchargement jusqu'à $3 \mathrm{kPa}$, de manière à évaluer I'influence de la contrainte verticale appliquée sur la dis. tribution de taille de pores. La confrotntation entre les distributions après compectage, après gonflement libre et après humidification sous $200 \mathrm{kPa}$ (Fig. 14) montre: - que l'humidification a conduit à une augmentation du diamëtre médian et du volume total des micropores. Ce phéthomène correspondrait a gonflement des agrégats

- que J'humidification génère une diminution du volume inter-agrégats d'autant plus importante que la contrainte verticale est élevée, ce qui est cohérent avec l'interprétation des essais d'humidification sous contrainte sur l'argile AvA34 [cf. \$ 4.2).

\section{6}

\section{Conclusion}

La demarche adoptée pour cette recherche a consisté à proposer un modèle de microstructure des sols fins compactés non saturés, sur la base des connaissances actuelles et gräce à de nouvelles observations en microscopie électronique environrementale. Ce modèle permet de quantifier deux paramétres microstructuraux gräce à des essais géotechriques conventionnels. Son utilisation pour exploiter et interpreter les essais de gonflement libre el d'humidifcation sous contrainte permet de décrire l'évolution du volume des vides intra-agrégats et inter-agrégats au cours des phénonènes liés à l'humidification du sol. Ces preniers résultats semblent confirmer que la part réversible du phénomène devrait être associée au comportement des agrégats, dont le volume semble essentiellement gouverne par la teneur en eau, alors que la part irréversible résulte plutôt des évolutions du volume des vides inter-agrẻgats, ce qu"avalent suggéré Alonso et al. (1999).

Cette approche a aussi permis de décrire distinctement l"influence de l'êtat initial (teneur en eau et masso

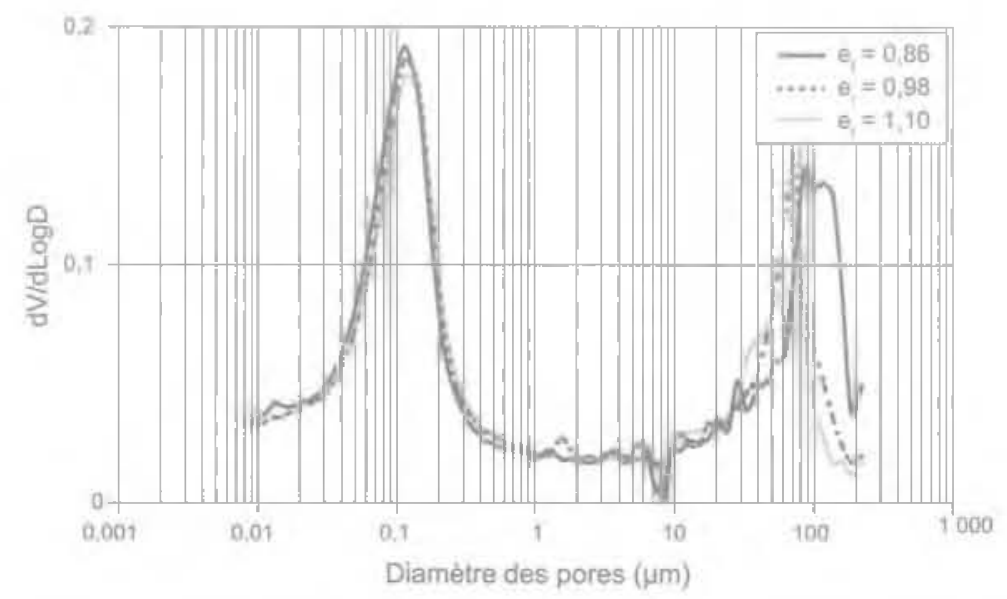




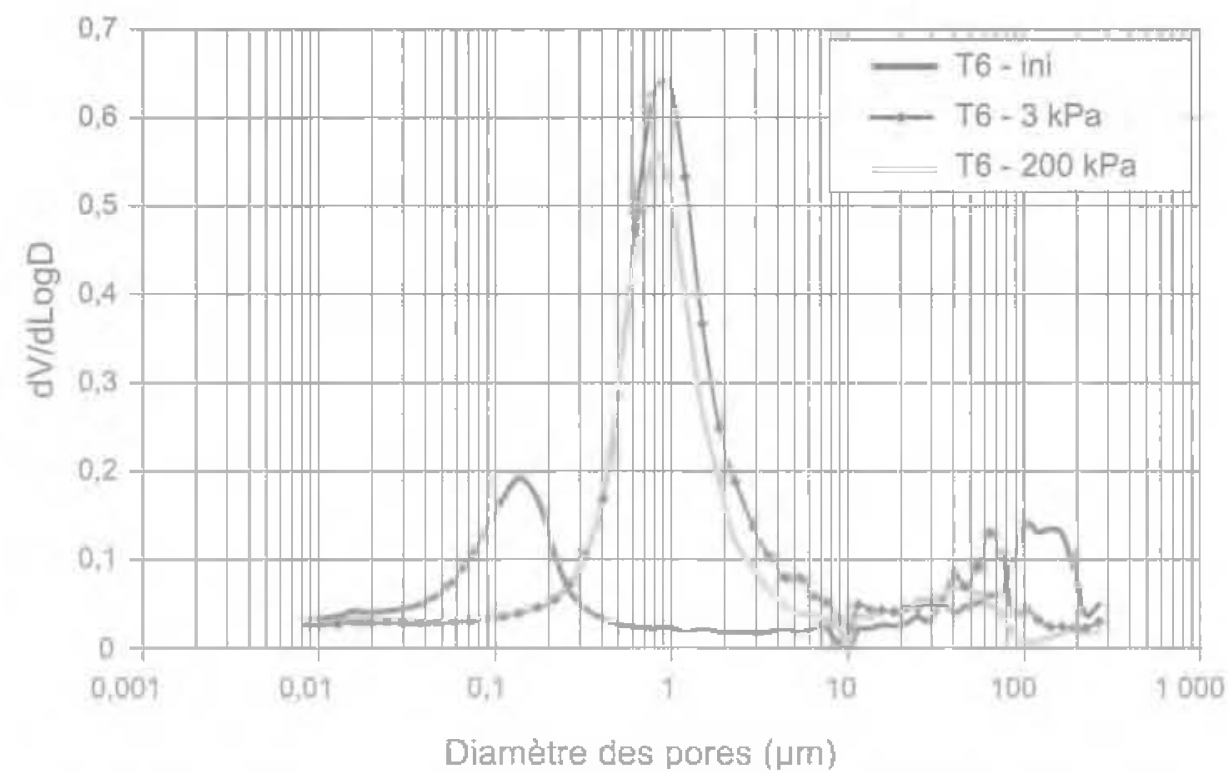

Fin 14 Influence de la sollicitation sur la distribution des tailles de pores (argile Av34, indice des vides initial ; 1, 1).

volumique sèche), de la nature du sol et la contrainte verticale sur les déformations. II en résulte une corrélation intéressante avec la limite de liquidité pour le gonflement libre et une description des phéromènes à l'échelle microscopique en général. La confrontation avec les analyses par porosimétrie par intrusion de mercure semble donner des arguments lavorables aux interprétations proposées, même si des expérimentations complémentaires sur d'autres sols et à partir d'autres états initiaux mériteraient d'être faites.

En outre, la méthodologie développée dans le cadre de cette recherche devrait pouvoin être appliquée au phénomène du retrait, ce qui permettrait de décrire de manière plus complète les défomations par variations d'état hydrique. Par suite, les risclues de retrait-gonflement devraient pouvoir être mielx appréciés gräce à des essais d'identification simples mais dont la pertinence vis-à-vis du phénomène aura pu être démontrée.

Enfin, les résultats de cette recherche permettent de proposer de nouvelles méthodes de conception des remblais routiers et ferroviaires, en perticulier dans les contextes sensibles (remblais de grande hauteur. remblais en zone inondable...) pour lesquelles aucune méthode rigoureusement justifiée n'est actuellement disponible. 
Alonso E.E., Vaunat J., Gens A. - Modelling the mechanical behaviour of expanslve clays. Engineering Geology, vol. 54 $n^{\circ} 2.1999$, p. 173-183.

Benett R.H., Hubert M.H. - Clay microstructure, Intemational human resources development corportation. Boston. 1980, $18.1 \mathrm{p}$

Bird N. Pertier E. - The pore-solid frac. tal model of soil density scaling. European Journal of Soil Science, 54 (3), 2003. p. $467-476$.

Boivin P. Garnier P. Tessier D. - Rela. tionship between clay content, clay type and strinkage properties of soil sam. ples. Soil Sci. Soc. Am. J., 68. 2004, p. 1.145-1153.

Braudeau E. - Équation généralisée des courbes de retrajt d'échantillons de sols structurés. C.R. Acad. Sci. Ser. 2, 307. 1988, p. 1731-1734.

Bruand A., Prost R. - Effect of water conent on the fabric of a soil material: An experimental approacli. European Joumal of Soil Science. 38 (3). 1997, p. $461-472$.

Delage P. Audiguier M., Cui Y.J., Howat M.D. - Microstructure of a compacted silt. Canadian Geotechnical Journal, vol. $33, \pi^{\circ} 1,1996$, p. 150-158.
Dertiche Z., Kebaiti M. - Prévision du gonflement des argiles d'in-Amémas. Bullefir des laboratores des ponth ep chaussées, $\pi^{2}$ 218, 1998, p. 15-23.

Diamond S. - Pore size distributions in clavs. Clays and clay minerals, vol. 18, $1969,0.7-23$

Gens A., Alorso E.E., Suriol J. - Effect of structure an the volumetric behaviour of a compacted soil. Proc. of the 15 international conference on unsaturated soils, UNSAT'D5. Paris, France, 6-8 sept. 1995, p. 83-88.

Gimenez D.. Allmaras F. R., Nater E.A. Huggins D.R. - Fractal dimensions for volume and surface of interaggregate pores: Scale effects. Geoderna. vol. 77 , [1.1997, p. 19-38:

Holtz W.G., Gibbs H.J. - Engineering properties of expansive clays. Trans. ASCE. vol. $121,1956,0,641-663$.

Lambe T.W - The structure of compacied clay. J. Geotech. Erong and Found. Div. ASCE, vol. 84. $n^{\circ} 2$ 2, paper 1654, 1958, p. $1654: 35$

Lawton E.C. Fragasy R.J. Hardcastle 3.H. - Collapse of compacted clayey sand. Journat of Geotechnical Engineering, vol. 115, 17 9. 1989, p. 1252-1267.

Pellerin FM. - La porosimétrie au mercure apoliguée ả l'étude géotechnique des sols et des roches. Bulletin des iaboratojres des ponts et chaussées, no 106,1980 , p. $105-116$

Seed H.B. Woodward Fi, J. Lurdgren R. Prediction of swelling potential for compacted clays. Joumal of the sol' mechaMics and foundation division. ASCE, SM3, 1962, p. 53.87.

Serratrice J.F. Soyez B. - Les essais de gonflement. Bulletin des laboratoires des ponts et chaussées. $n^{5} 204,1996$. p. $65-85$

SETRA-LCPC - Rérilsation des remblais et des couches de forme [GTR!. Guide technique SETRA-LCPC, 2 fascicules. 1992,98 el $102 \mathrm{p}$.

Tessier D. - Etude expérimentate de l'organisation des matériaux argileux. Hydratation, gonflement et structurafion all cours de la dessictation et de la réhumectation. INRA, these de doctorat ès sciences de l'universite de Paris VTi. 1984, $362 \mathrm{p}$.

Wan A.W., Gray M.N., Grahan J. - On the relations of suction, moisture content and soils structure ín compacted clays. Proc. of the ist international conference on unsaturated soils, UNSAT'95, Paris, France, 6-8 sept. 1995, p. 215-222. 